\title{
Defect Analysis of Sputter Grown Cupric Oxide for Optical and
}

\section{Electronics Application}

\author{
Goutam Kumar Dalapati, ${ }^{1 *}$ Rasanayagam Sivasayan Kajen, ${ }^{1}$ Saeid Masudy-Panah, ${ }^{1}$ and \\ Prashant Sonar ${ }^{1,2}$ \\ ${ }^{1}$ Institute of Materials Research and Engineering, A*STAR (Agency for Science, Technology \\ and Research), 3 Research Link, 117602 Singapore. \\ ${ }^{2}$ School of Chemistry, Physics and Mechanical Engineering, Queensland University of \\ Technology, GPO Box 2434, Brisbane QLD 4001, Australia \\ Corresponding author: \\ *G. K. Dalapati: dalapatig@imre.a-star.edu.sg
}

\begin{abstract}
:
We have studied the defect density and defect level of sputter grown cupric oxide $(\mathrm{CuO})$ for optical and electronic applications. Deep Level Transient Spectroscopy (DLTS) technique has been employed to study the defect density in the cupric oxide thin film deposited by sputtering. The DLTS studied showed that the defects density significantly reduced for the film grown at high working pressure. It has been also shown that by doping, defect density increases for the film grown at high working pressure. High-resolution TEM analysis has also shown the improvement in a crystallinity of samples prepared at high working pressure. The optical characteristics show that the sputter grown $\mathrm{CuO}$ exhibits a bandgap of $1.4 \mathrm{eV}$ with absorption coefficient of $\sim 10^{4} \mathrm{~cm}^{-1}$. From photo electron spectroscopy measurement, it was found that the work function for $\mathrm{CuO}$ is $\sim 5.2 \mathrm{eV}$. The present work reveals the importance of $\mathrm{CuO}$ for optical and electronic device applications.
\end{abstract}




\section{Introduction:}

Metal oxides have proved to be as promising classes of materials for the optical and electronic device applications [1]. In the literature, majority of the work has been done on n-type metal oxides, their conductivity and its various applications in optoelectronics [2-3]. There are a very few metal oxides reported so far which exhibits $p$-type conductivity and the research on this direction is extremely useful in order explore the library of $p$-type materials. Among them, cupric oxide $(\mathrm{CuO})$ is one of the potential materials for solar cells and electronic applications. $\mathrm{CuO}$ exhibits a bandgap of $\sim 1.4 \mathrm{eV}$, which is an ideal for solar cell application and better photon absorption. An appropriate band gap of $\sim 1.4$, better optical absorption and its plenty of abundance with cheap cost make this vividly explored material as an ideal choice for the solar absorption and photovoltaic technology [4-6].

The maximum power conversion efficiency (PCE) reported using $\mathrm{CuO}$ based single junction solar cell is around 1\% [4-6], which is far from theoretically predicted PCE value of $31 \%$ [7]. The difficulties of growing $\mathrm{CuO}$ with high hole density and less defects are some of the main and important challenging parameters for the poor performance of $\mathrm{CuO}$ based solar cells. An understanding of the defect formation and finding method to increase carrier density of $\mathrm{CuO}$ is therefore crucial to improve the performance of $\mathrm{CuO}$ based solar cell devices.

In $\mathrm{CuO}$ based solar cells, a large number of various types of intrinsic defects are possible in the structure. Among these defects, defects related with deep-level are responsible for the recombination which limits the power conversion efficiency (PCE) due to reduction in open circuit voltage $\left(V_{o c}\right)$. A deep level defect not only reduces the $V_{o c}$ but it may hamper the charge collection efficiency to the respective electrodes arising from the thin film characteristics of the 
solar cells attributed with their charges and its recombination. Therefore, gaining knowledge about the defect characterization, defect energies and their densities have a paramount importance for designing high performance $\mathrm{CuO}$ based photovoltaic devices and improving its solar cell efficiency. Information about the characteristics of deep level defects in a semiconductor can be obtained using a novel Deep Level Transient Spectroscopy (DLTS) characterization technique [8]. DLTS is a useful technique for the investigation of electrical defect states and is a powerful diagnostic tool for the identification of deep defect levels in $p-n$ junctions and Schottky barriers. It is also useful to characterize deep states in active semiconductor thin films and the process induced defect states in heterojunction solar cells [9]. Furthermore, it can be used to determine the trap properties such as trap energy level, capture cross section, and trap concentration [10-11]. However, till to date, there is no research conducted about the systematic analysis of deep level defects of thin film $\mathrm{CuO}$. These defects and their investigation are thought to be of high importance in order to produce high efficiency solar cells. In this work, DLTS technique has been used as an electrical defect characterization method to get information about the trap states in the $\mathrm{CuO}$ absorbers thin film and at the $p$ $\mathrm{CuO} / n$-Si heterojunction interface. An influence of the sputtering working pressure on defects states, crystal quality, optical properties and surface morphology of $\mathrm{CuO}$ were also investigated by using high resolution transmission electron microscopy (HR-TEM), UV-VIS Spectrophotometer, and atomic force microscopy (AFM) techniques, respectively. 


\section{Experiment:}

$\mathrm{CuO}$ thin films were grown at room temperature by using Radio-Frequency (RF) magnetron sputtering with stochiometric $\mathrm{CuO}$ target. $\mathrm{Si}$ and glass were used as the substrates for $\mathrm{CuO}$ deposition. $\mathrm{CuO}$ was deposited at two different working pressures of 3.3 mTorr and 30 mTorr. Samples were thermally treated at $300^{\circ} \mathrm{C}$ for 1 min in nitrogen ambient by using the rapid thermal annealing system. The thickness of the $\mathrm{CuO}$ films grown on $\mathrm{Si}$ and glass substrate was $150 \mathrm{~nm}$. The $\mathrm{CuO}$ thin film surface morphology was analyzed by using AFM (Bruker Dimension Icon atomic), and the crystallinity of the $\mathrm{CuO}$ was examined using HR-TEM (Philips CM300). Bilayer of titanium (Ti) and Aluminum (Al) was deposited by sputter on $\mathrm{CuO}$ and backside of $n$ silicon to make Ohmic contact for DLTS measurement. Figure 1 shows the schematic diagram of the device. Capacitance-Voltage and DLTS measurements were performed using BIO-RAD's DL 8000 Fourier DLTS system at a frequency of 1 MHZ. Fourier DLTS measurements were carried out using a reverse bias voltage of $-3 \mathrm{~V}$, pulse voltage of $-0.1 \mathrm{~V}$ and a pulse width of $1 \mathrm{~s}$. 


\section{Result and Discussion:}

We have studied the defect density and defect level of sputter grown $\mathrm{CuO}$ thin films using DLTS technique. A significant DLTS peak, $\mathrm{E}_{1}$ is observed from our DLTS measurements for both the samples grown at 3.3 mTorr [Lower Pressure (LP)] and 30 mTorr [Higher Pressure (HP)], as shown in Fig 1(a). The peaks are corresponding to a trap energy level of $1.1 \mathrm{eV}$ extracted from the Arrhenius analysis (Fig. 2(b)). The trap density to be significantly lower for the device fabricated at HP (and $\left.1.1 \times 10^{14} \mathrm{~cm}^{-3}\right)$ as compared to the device fabricated at LP $\left(4.5 \times 10^{14} \mathrm{~cm}^{-}\right.$ ${ }^{3}$ ) which is might be due to the better thin film quality at HP in comparison with thin film processed at LP. In addition, from $C$ - $V$ measurements (see Fig. 3), we noticed a higher doping density $\left(1.25 \times 10^{15} \mathrm{~cm}^{-3}\right)$ is observed (approximately twice) for the HP device as compared to the LP device $\left(6 \times 10^{14} \mathrm{~cm}^{-3}\right)$ at $300 \mathrm{~K}$. While the extracted doping concentration usually includes both trapped charges and free charges, the trap densities extracted from our DLTS measurements indicate that a higher fraction of charges are trapped in the LP devices. Hence, we infer that the charge transport will be more effective in the HP devices due to a lowered resistivity (higher carrier concentration) and a smaller extent of trapping as compared to the LP devices.

Effect of working pressure on the grain growth and crystal quality of prepared samples is investigated by high-resolution TEM (HR-TEM). Figure 4 shows HR-TEM images of sputter deposited thin films at low and high working pressure. As shown at Fig. 4, with increasing the working pressure the crystal quality improves. This improvement of crystal quality is the main reason for the reduction in defects density and increasing doping density for the film grown at high working pressure, which has been already studied and confirmed by DLTS study. 
The impact of working pressure on the surface morphology and grain height distribution was also studied by Atomic Force Microscopy (AFM) analysis. Fig. 5 shows AFM images and grain height distribution of prepared thin films as a function of working pressure. The grain height of sputtered thin films slightly increases as the working pressure is increased from 3.3 mTorr to 30 mTorr. The increase of grain height is might be due to the decrease of compressive stress. The decrease of tensile stress is caused by less oxygen defects in the lattice of the samples prepared at high working pressure. Less oxygen defects in the lattice can promotes the crystallinity of deposited thin films and increase grain size.

Fig. 6(a) shows the optical absorption coefficient of sputter grown $\mathrm{CuO}$. There are no significant changes in the absorption property of $\mathrm{CuO}$ grown at different working pressure. The optical absorption coefficient is $\sim 2 \times 10^{4} \mathrm{~cm}^{-4}$ at $1.4 \mathrm{eV}$. To determine the optical bandgap, Tauc curves of $(\alpha h v)^{0.5}$ versus photon energy $(h v)$ are plotted at Fig. 6(b), where $\alpha$ is the absorption coefficient. The optical bandgap $\left(E_{g}\right)$ is estimated from the intercept of $h v$ axis with extrapolated linear segments of the Tauc curve. The extracted optical bandgap of $\mathrm{CuO}$ is around $1.4 \mathrm{eV}$ which is in a well agreement with reported results by Dimopoulos et al [3]. It is worth to note that the by tuning the working pressure during sputter deposition, it is possible to improve the crystal quality and doping concentration without change in optical properties. Generally, if the phase of the copper oxide changes, it significantly influenced on the optical properties. In order to determine the work function of $\mathrm{CuO}$ deposited thin film on $\mathrm{n}-\mathrm{Si}(100)$ substrate, photoelectron spectroscopy in air (PESA) measurements were carried out. The photoelectron output and applied UV energy is plotted with horizontal X-axis and the vertical Y-axis respectively. The slope of graph $(\mathrm{Y} / \mathrm{eV})$ determines the standardized photoelectron yield ratio. The work function 
value of $\mathrm{CuO}$ thin film deposited via $\mathrm{RF}$ sputtering and annealed at $300{ }^{\circ} \mathrm{C}$ was calculated from the onset energy level recorded in PESA shown in Figure 7, which is found to be $5.2 \mathrm{eV}$ [12].

\section{Conclusion:}

Sputter grown $\mathrm{CuO}$ has been studied for the optical and electrical applications. The defect density and defect level of sputter grown $\mathrm{CuO}$ was investigated as a function of working pressure. We believe that the higher pressure of the sputtering chambers allows minimal time to allow the defect formation whereas in the lower pressure the growth rate is limited and there would be propensity to form more defects. It was shown that electrical properties and defects density can be controlled through sputter deposition. The film grown at high working pressure reduced the crystal defects without change of optical properties. It was also found that defect level can be reduced and carrier density can be increased by sputter depositing at high working pressure. The work function for $\mathrm{CuO}$ deposited on $n$-Si(100) structure after annealed at $300{ }^{\circ} \mathrm{C}$ was found to be $5.2 \mathrm{eV}$ indicate the appropriate energy level for the charge extraction. 


\section{References:}

${ }^{1}$ S. Rühle, A. Y. Anderson, H-N. Barad, B. Kupfer, Y. Bouhadana, E. Rosh-Hodesh, A. Zaban, J. Phys. Chem. Lett. 3, 3755 (2012).

${ }^{2}$ R. Jose, V. Thavasi, S. Ramakrishna, J. Am. Ceram. Soc. 92, 289 (2009).

${ }^{3}$ Y. S. Lee, J. Heo, M. T. Winkler, S. C. Siah, S. B. Kim, R. G. Gordon, T. Buonassisi, J. Mater. Chem. A 1, 15416 (2013).

${ }^{4}$ F. Gao, X-J Liu, J-S Zhang, M-Z Song, N Li, J. Appl. Phys. 111, 084507 (2012).

${ }^{5}$ S. Masudy-Panah, G. K. Dalapati, K. Radhakrishnan, A. Kumar, H. R. Tan, J. Appl. Phys. 116, 074501 (2014).

${ }^{6}$ S. Masudy-Panah, G. K. Dalapati, K. Radhakrishnan, A. Kumar, H. R. Tan, E. N. Kumar, C. Vijila, C. C. Tan, D. Z. Chi, Prog. Photovolt: Res. Appl.. doi: 10.1002/pip.2483

${ }^{7}$ Y. Peng, Z. Zhang, T.V. Pham, Y. Zhao, P. Wu, J. Appl. Phys. 111, 103708 (2012).

${ }^{8}$ D. V. Lang, J. Appl. Phys. 45, 3023 (1974).

${ }^{9}$ J. Van Gheluwe, P. Clauws, Thin Solid Films 515, 6256 (2007).

${ }^{10}$ R. N. Bhattacharya, A. Balcioglu, K. Ramanathan, Thin Solid Films 384, 65 (2001).

${ }^{11}$ G. K. Paul, Y. Nawa, H. Sato, T. Sakurai and K. Akimoto, Appl. Phys. Lett. 88, 141901 (2006).

${ }^{12}$ G. K. Dalapati, A. Kumar, C. C. Tan, S. L. Liew, P. Sonar, H. L. Seng, H. K. Hui, S. Tripathy, and D. Z. Chi, ACS Appl. Mater. Interfaces 5, 5455 (2013). 


\section{Figure captions:}

Figure 1: Schematic diagram of $p-\mathrm{CuO} / n$-Si heterojunction for DLTS study.

Figure 2: a) DLTS spectra for devices with $\mathrm{CuO}$ grown at low pressure (blue curve with squares) and high pressure (purple curve with circles) respectively. While both devices show a common trap level at $1.1 \mathrm{eV}$ from Arrhenius plots (see Fig. 2(b)), the extracted trap density is found to be larger in the LP device as compared to the HP device. b) Arrhenius plots for both HP ( purple line with triangles) and LP devices (blue line with squares).

Figure 3: The doping concentration in the HP device (purple line with triangles) and the LP device (blue line with squares) as a function of temperature.

Figure 4: TEM images of the $\mathrm{CuO}$ thin films prepared by sputter deposition at (a) high and (b) low working pressure.

Figure 5: (a) AFM images and (b) grain height distribution of the $\mathrm{CuO}$ thin films prepared by sputter deposition at low and high working pressure.

Figure 6: (a) Optical absorption coefficient of thin films prepared by sputter deposition at low and high working pressure. (b) Plot of $(\alpha h v)^{0.5}$ versus photon energy $(h v)$.

Figure 7: Photoelectron spectrum of $\mathrm{CuO} / n-\mathrm{Si}(100)$ structure after annealed at $300{ }^{\circ} \mathrm{C}$. 


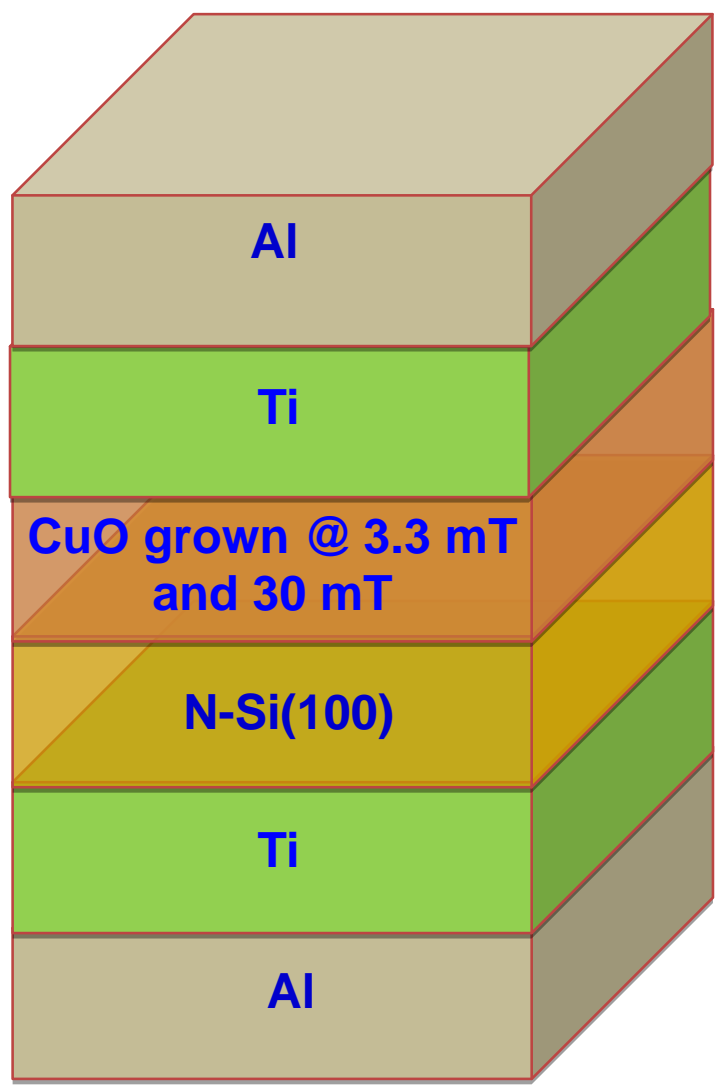

Figure 1: Schematic diagram of $p-\mathrm{CuO} / n-\mathrm{Si}$ heterojunction for DLTS study 


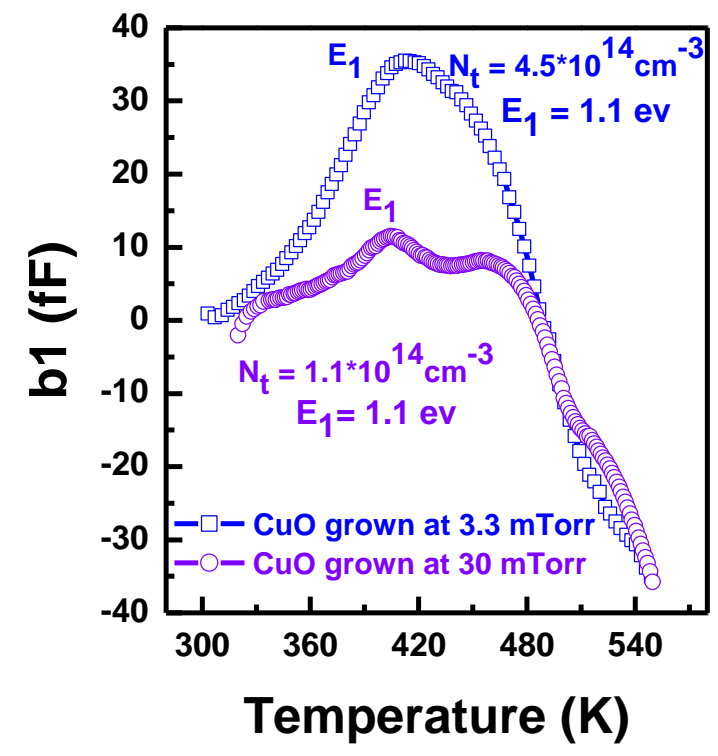

(a)

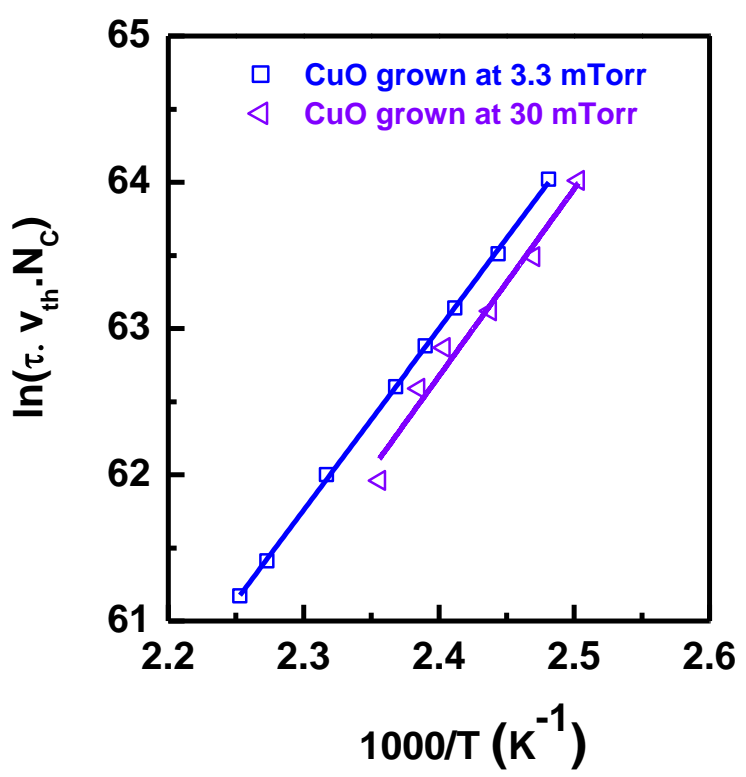

(b)

Figure 2: a) DLTS spectra for devices with $\mathrm{CuO}$ grown at low pressure (blue curve with squares) and high pressure (purple curve with circles) respectively. While both devices show a common trap level at $1.1 \mathrm{eV}$ from Arrhenius plots (see Fig. 2b), the extracted trap density is found to be larger in the LP device as compared to the HP device. b) Arrhenius plots for both HP ( purple line with triangles) and LP devices (blue line with squares). 


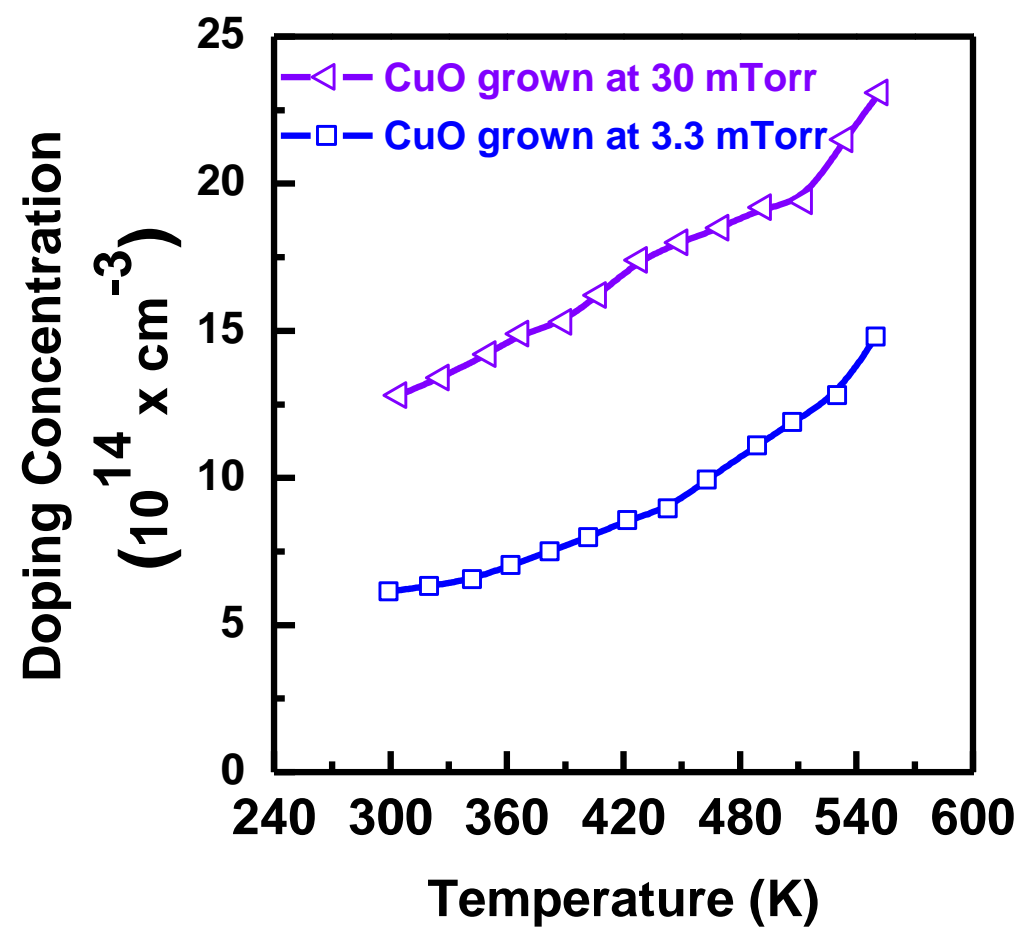

Figure 3: The doping concentration in the HP device (purple line with triangles) and the LP device (blue line with squares) as a function of temperature. 

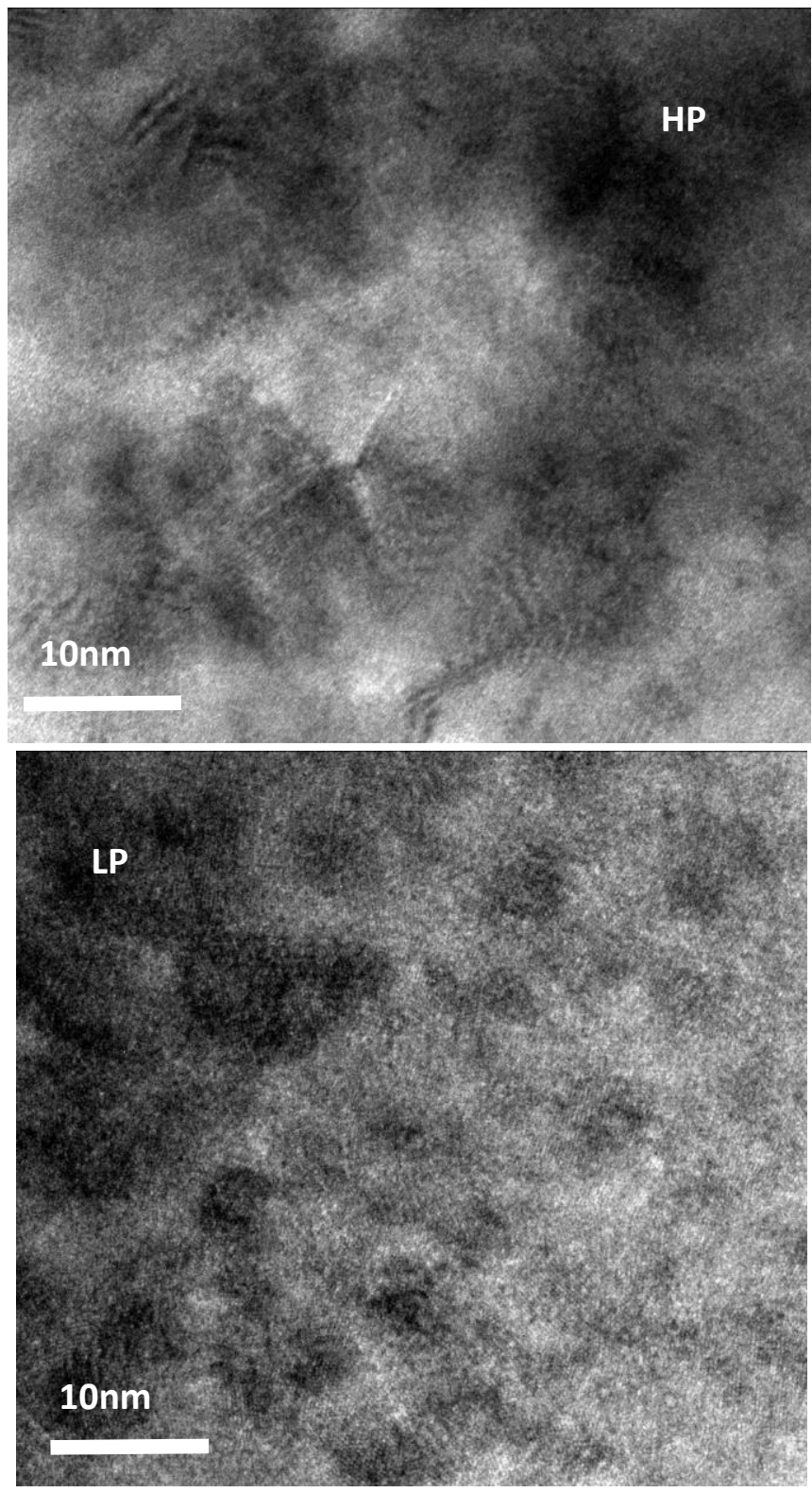

Figure 4: TEM images of the $\mathrm{CuO}$ thin films prepared by sputter deposition at (a) high and (b) low working pressure. 


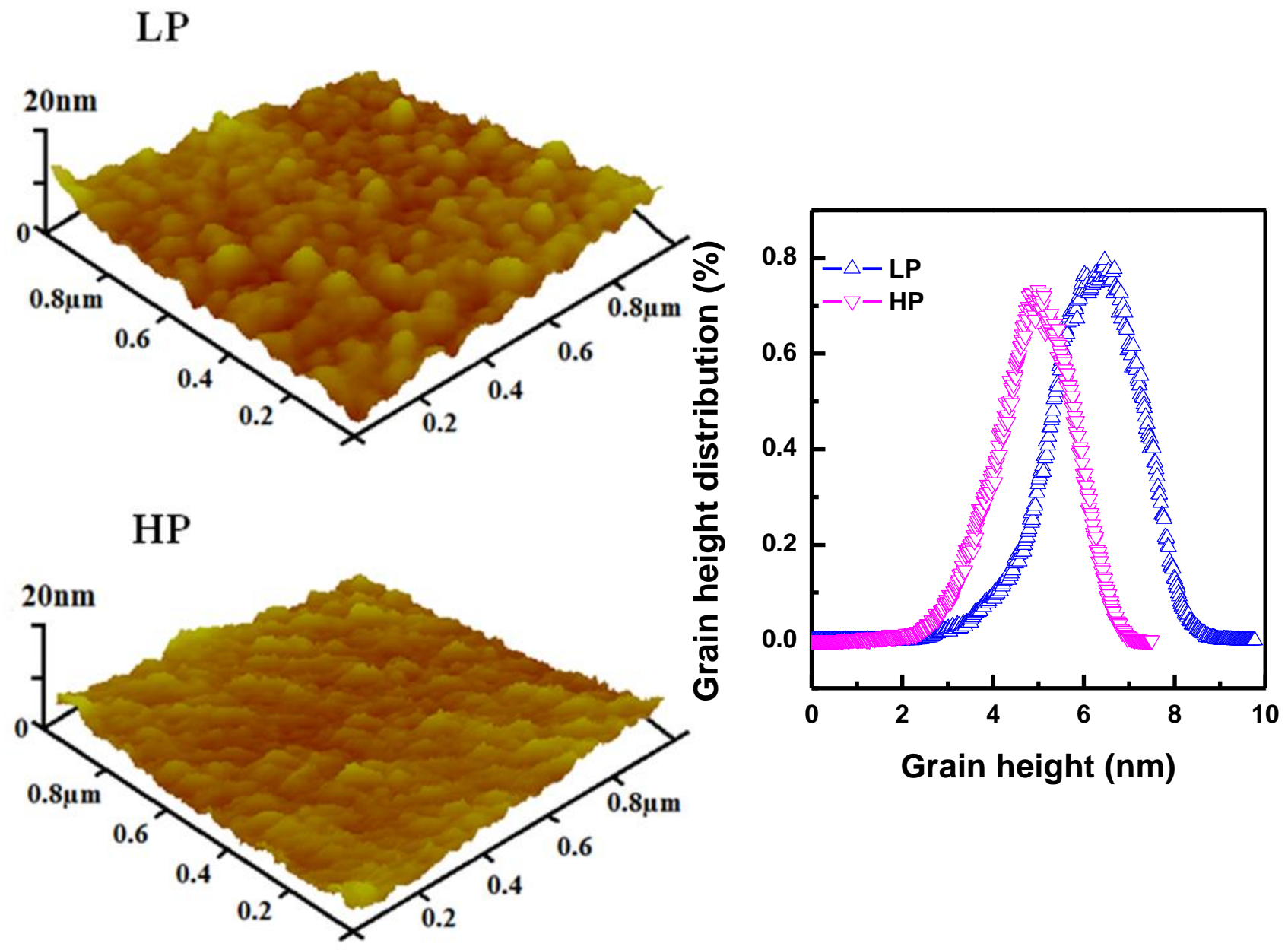

Figure 5: (a) AFM images and (b) grain height distribution of the $\mathrm{CuO}$ thin films prepared by sputter deposition at low and high working pressure. 

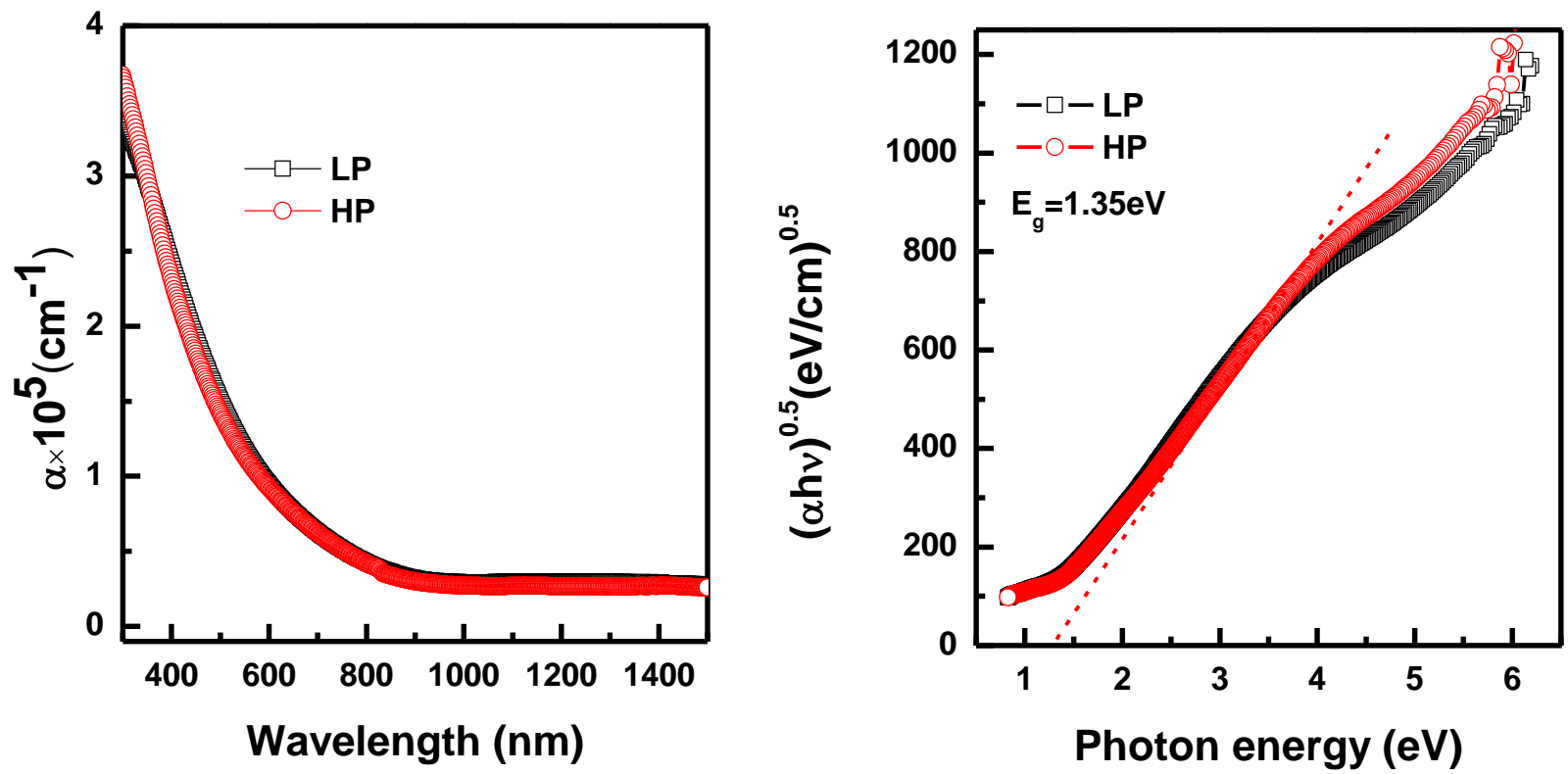

Figure 6: (a) Optical absorption coefficient of thin films prepared by sputter deposition at low and high working pressure. (b) Plot of $(\alpha h v)^{0.5}$ versus photon energy $(h v)$. 


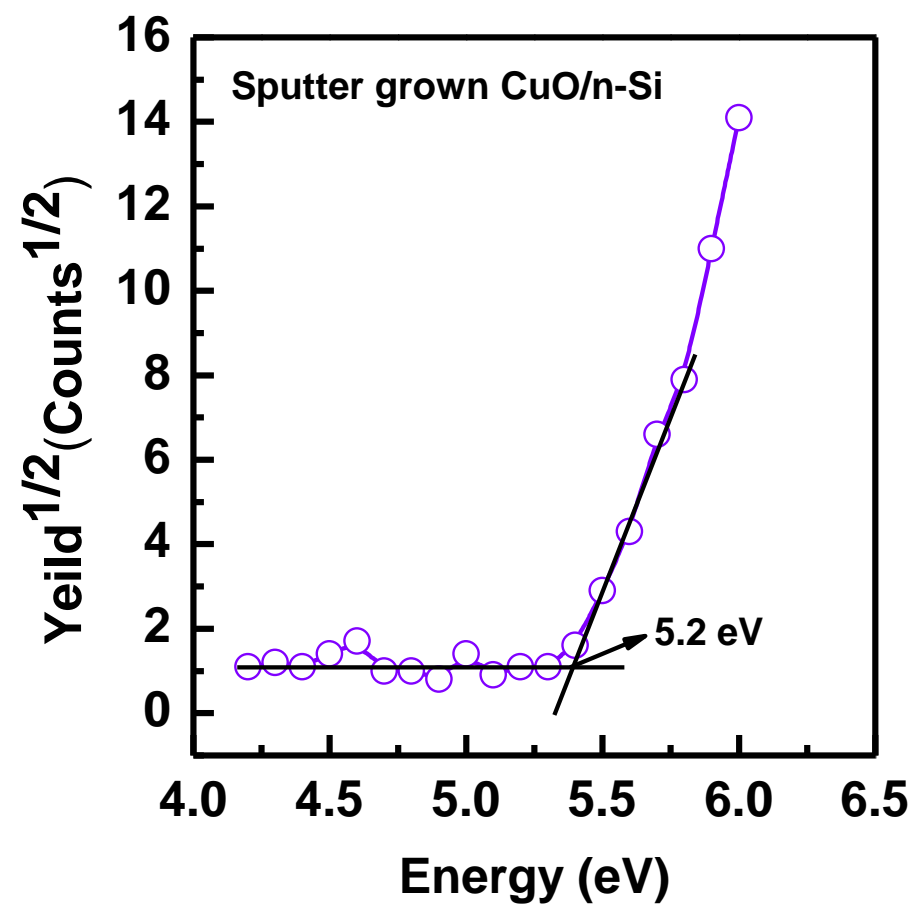

Figure 7: Photoelectron spectrum of $\mathrm{CuO} / n-\mathrm{Si}(100)$ structure after annealed at $300{ }^{\circ} \mathrm{C}$. 